\title{
Non-Hermitian Sonic Second-Order Topological Insulator
}

\author{
Zhiwang Zhang, ${ }^{1,2}$ María Rosendo López, ${ }^{2}$ Ying Cheng, ${ }^{1, *}$ Xiaojun Liu, ${ }^{1, \dagger}$ and Johan Christensen ${ }^{2, \$}$ \\ ${ }^{1}$ Department of Physics, MOE Key Laboratory of Modern Acoustics, Collaborative Innovation Center of Advanced Microstructures, \\ Nanjing University, Nanjing 210093, China \\ ${ }^{2}$ Department of Physics, Universidad Carlos III de Madrid, ES-28916 Leganés, Madrid, Spain
}

(Received 7 January 2019; published 15 May 2019)

\begin{abstract}
Topological phases of matter that have been recently extended to topological phases of sound can confine acoustic energy at the corners of higher-order topological insulators. We broaden this concept by incorporating parity-time symmetry and show new topologically protected confinement rules that are dictated by the geometrical arrangement of gain and loss units. Particularly, our findings reveal how sound trapping occurs at all corners when parity-time symmetry is intact, beyond the exceptional point within the broken phase; however, opposite corners sustain either sink- or sourcelike states that could lead to novel non-Hermitian guides for sound.
\end{abstract}

DOI: 10.1103/PhysRevLett.122.195501

Topological insulators (TIs) hosting robust gapless edge states have paved an exciting way towards novel wave engineering in condensed matter physics [1-3], which also stimulated the search for analogous states in classical systems such as photonics [4-15], acoustics [16-27], and mechanics [28-30]. Recently, this concept was generalized to higher-order TIs (HOTIs) belonging to a special class, in which the conventional bulk-boundary correspondence does not apply [31-37]. So far, HOTIs have been experimentally reported in 2D elastic [38], microwave [39], electric circuit [40], and 3D acoustic systems [41,42].

On the other hand, parity-time $(\mathcal{P} \mathcal{T})$ symmetric systems are a special kind of physical configuration that is invariant upon the combined operations of parity inversion $\mathcal{P}$ and time reversal $\mathcal{T}$ [43-46]. The eigenvalues of the $\mathcal{P} \mathcal{T}$ symmetric Hamiltonian are real as long as the nonHermitian parameter is below a specific threshold, which is called as the exceptional point (EP). When tuning beyond the EP one is able to break the $\mathcal{P} \mathcal{T}$ symmetry at which the eigenvalues become complex. Within this $\mathcal{P} \mathcal{T}$-broken phase, wave propagation asymmetry sets in that can lead to one-way cloaks and asymmetric reflections as has been explored for both optical [47-50] and acoustical [51-56] systems.

In this Letter, we propose a 2D second-order topological sonic crystal (SC) and study both its Hermitian and nonHermitian acoustic characteristics. We construct a concentric square crystal (CSC) whose basic building blocks are fluidic SCs. By exploring the signatures of this HOTI, we unravel novel complex pseudohinge and corner states through engineered $\mathcal{P} \mathcal{T}$ symmetric metamolecules. These metamolecules hosting cleverly balanced acoustic gain and loss components, dictate yet unseen topologically confined pseudohinge and corner states whose confinements vary across the $\mathcal{P} \mathcal{T}$ breaking transition.
We start with a regular Hermitian square SC as shown in Fig. 1(a), which is composed of isotropic cylinders embedded in air background. In the following, we define a metamolecule consisting of four neighboring cylinders as the primitive cell [see the inset of Fig. 1(a)]. The lattice constant is $a=1 \mathrm{~cm}$ and the radii of the cylinders are $r=0.12 a$. The distance between the adjacent cylinders is defined as $D$, which for the present regular SC is chosen to be $D / a=0.5$. The mass density and the bulk modulus of
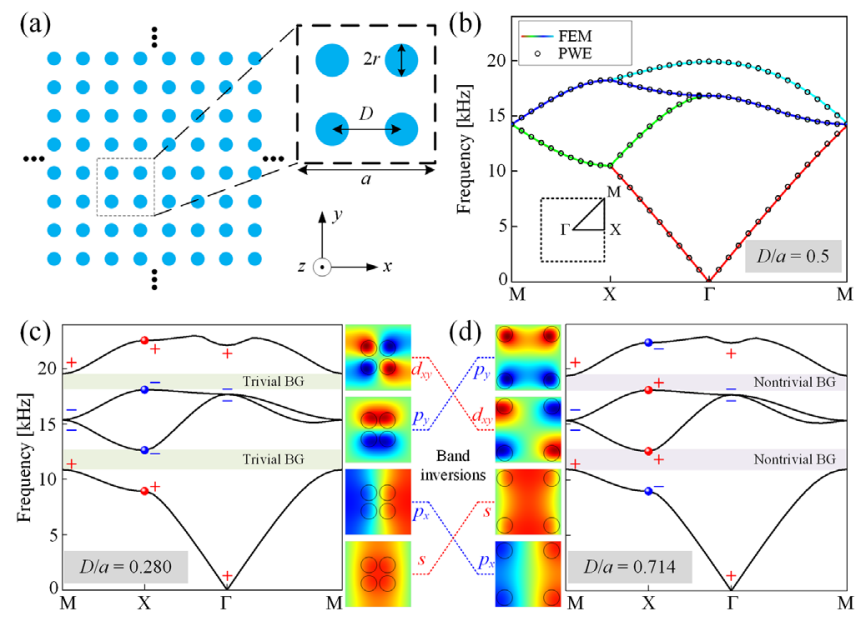

FIG. 1. (a) Schematic illustration of an unperturbed fluid SC. (b) Dispersion relation for a structure with ratio $D / a=0.5$. Colored curves display the results calculated with the FEM and the circles represent the bands obtained by the PWE method. Inset: the first Brillouin zone (BZ). Band inversions induced by tuning the ratio from (c) $D / a<0.5$ (shrunk metamolecule) to (d) $D / a>0.5$ (expanded metamolecule). The "+/-" signs represent the even and odd parity at the high symmetry points before and after the band inversions. Insets: eigenmodes at the $X$ point from the lowest to the highest band. 
air are $\rho_{0}=1.21 \mathrm{~kg} / \mathrm{m}^{3}$ and $\kappa_{0}=1.4 \times 10^{5} \mathrm{~Pa}$, respectively. Contrary, the mass density and the bulk modulus of cylinders are $\rho=\rho_{0}$ and $\kappa=\kappa_{0} / 9$, respectively. Figure 1(b) shows the dispersion relations for the aforementioned regular SC, obtained by the plane wave expansion (PWE) technique [57] and a finite-element method (FEM). A topological phase transition is introduced by either shrinking the metamolecule $(D / a<0.5)$ or expanding it $(D / a>0.5)$ as shown in Figs. 1(c) and 1(d), respectively. When $D / a<0.5$, the modal distributions of the four eigenmodes at the $X$ point, from bottom to top, are characterized as $s$, a pair of $p$ and $d_{x y}$ acoustic modes [20,58]. The degenerated $p$ modes have an odd parity (-), while the isolated $s$ and $d_{x y}$ modes have an even parity $(+)$. The parities at high symmetric points are marked as " \pm " in Fig. 1(c). Contrary, for the expanded counterpart, $D / a>0.5$, the parities near the band gaps at the $X$ point are inverted as shown in Fig. 1(d), which indicates a topological phase transition. This topological phase transition can be characterized by the vectorial Zak phase, which is the integration of the Berry connection over the momentum space, [59]

$$
\mathbf{P}=\iint_{1 \mathrm{stBZ}} d k_{1} d k_{2} \operatorname{Tr}(\mathbf{A}), \quad \mathbf{A}_{\mathrm{mn}}=i\left\langle u_{\mathrm{m}}\left|\partial_{\mathbf{k}}\right| u_{\mathrm{n}}\right\rangle,
$$

in which $\left|u_{\mathrm{m}}\right\rangle$ is the Bloch function for the $m$ th band where $m, n$ run over occupied bands. Because of the $C_{4 v}$ point group symmetry, the Zak phase can be rewritten as $[58,59]$

$$
P_{i}=\pi\left(\sum_{n} q_{i}^{n} \text { modulo 2 }\right), \quad(-1)^{q_{i}^{n}}=\frac{\eta_{n}\left(\mathrm{X}_{i}\right)}{\eta_{n}(\Gamma)},
$$

where $i=x, y$ represents the direction, $X_{i}$ is the $X(Y)$ point of the first BZ, and $\eta_{n}$ is the parity of the $n$th band at the high symmetry point. It has been proven that the Zak phase is related to $2 \mathrm{D}$ polarization through $\sigma_{i}=P_{i} / 2 \pi$ with $i=x, y[60,61]$. Derived from Eqs. (1)-(2) and Figs. 1(c)$1(\mathrm{~d})$, the 2D Zak phase of the SC equals $(0,0)$ with $\boldsymbol{\sigma}=$ $(0,0)$ for $D / a<0.5$, which means that the SC is in the topological trivial phase. For $D / a>0.5$, the $2 \mathrm{D}$ Zak phase equals $(\pi, \pi)$ with $\boldsymbol{\sigma}=(1 / 2,1 / 2)$ and the SC is in the topological nontrivial phase. The quantized dipole bulk polarization with $\sigma_{x}=\{0,1 / 2\}$ in the $x$ direction leads to the existence of the edge states propagating along the $y$ direction and vice versa. Note that the nontrivial dipole moments in both directions ( $x$ direction and $y$ direction) coexist in our system, which means a quadrupole moment can be obtained from these two unequal dipole moments. Consequently, corner states appear as a double projection of the quadrupole tensor at corners, which can be defined as $[62,63]$

$$
Q^{\text {corner }}=4 \sigma_{x} \sigma_{y}
$$

As a result, the nonzero topological quadrupole moment $Q^{\text {corner }}$ is topologically protected in a hierarchy of the bulkedge and edge-corner correspondence with a nonzero Zak phase in both directions $\left(P_{i} \neq 0, i=x, y\right)$. The relationship among parities at high symmetry points, topological phases, and the ratio $D / a$ is discussed elsewhere [57].

We demonstrate that topological pseudohinge and corner states can be hosted in a CSC composed out of a $8 a \times 8 a$ nontrivial region with $D / a=0.714$ enclosed by a trivial region $(D / a=0.280)$ of thickness $14 a$; see Fig. 2 . The computed discrete complex eigenfrequencies that are illustrated in Figs. 2(a) and 2(b) display purely real confined states, with the exception of leaky complex bulk states at higher frequency. The two first edge state branches seen in Fig. 2(a), in between $11-11.5 \mathrm{kHz}$, provide two doubly degenerate pseudohinge states (labeled as $\mathrm{pH}_{1}, \mathrm{pH}_{2}, \mathrm{pH}_{3}$, and $\mathrm{pH}_{4}$ ), whose mode profiles are rendered in Fig. 2(c). Conventionally, 1D topological chiral hinge states can only exist in 3D second-order TIs [31,36]. However, unlike these ordinary edge states, the CSC sustains topological states that only confine along either the horizontal or the vertical hinges as shown in Fig. 2(c). Thus, we refer to them as pseudohinge states for the sake of discrimination [57]. The topological corner charge of the inner nontrivial region $Q^{\text {corner }}=1$ gives rise to single corner states in each corner. As predicted in Figs. 2(a)-2(b), we obtain four real eigenmodes whose profiles confine at the CSC's (off-) diagonal corners and at all four corners simultaneously, see
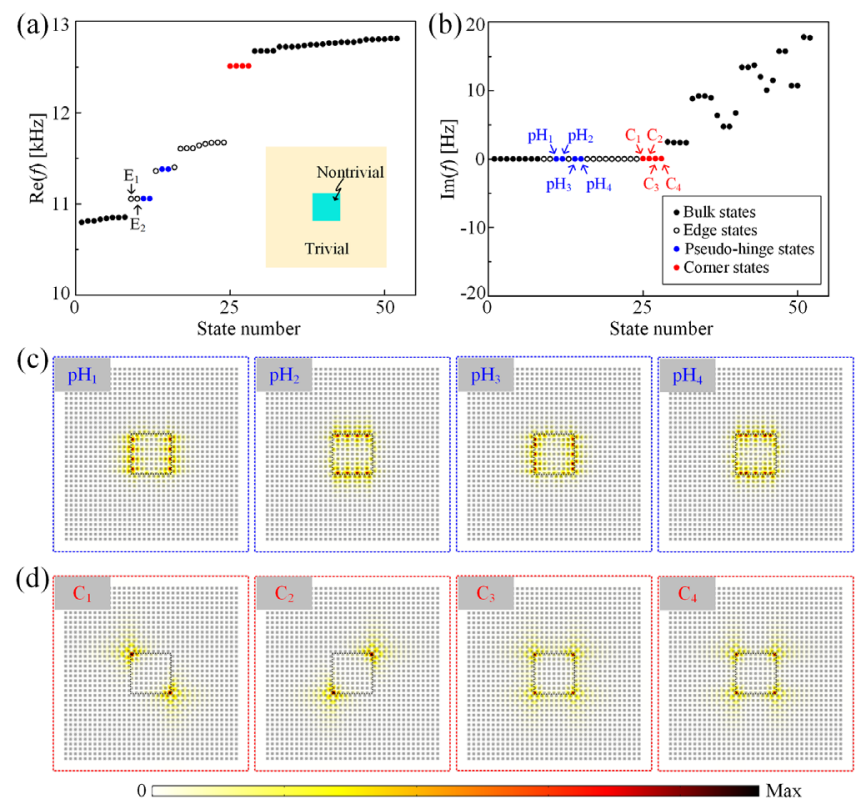

FIG. 2. Simulated (a) real and (b) imaginary eigenfrequencies of the Hermitian CSC. The crystal is made of a $8 a \times 8 a$ metamolecule array defining the nontrivial region with $D / a=0.714$, which is enclosed by a trivial region $(D / a=0.280)$ of thickness 14a. Black dots, circles, blue dots, and red dots denote the bulk, edge, pseudohinge and corner states, respectively. Acoustic eigenmode profiles of the (c) pseudohinge and (d) corner states. 
Fig. 2(d). Although we mainly focus on sound waves, second-order topological insulators in Hermitian systems that are characterized by the nontrivial 2D Zak phase can be readily constructed in other classical wave systems [62].

We extend the previous study of HOTIs in Hermitian CSCs to account for non-Hermitian ingredients in terms of acoustic loss and gain. In doing so, we employ an active acoustic metamaterials whose imaginary part of the effective mass density can be readily engineered as desired $[51,52]$. Thus, the resulting complex cylinder mass densities are defined as $\rho_{\text {Gain }}=(1+i \beta) \rho_{0}$ for the gain component and $\rho_{\text {Loss }}=(1-i \beta) \rho_{0}$ for the loss counterpart, while the bulk moduli remain, $\kappa=\kappa_{0} / 9$. The value of $\beta$ controls the amount of gain and loss. To balance out gain and loss within the metamolecule, two gain cylinders and two loss cylinders are considered for two specific arrangements.

As illustrated in Fig. 3 we construct a CSC similar to the previous Hermitian case study. Now however, metamolecules constitute the basic building blocks in which the nonHermitian components have been introduced parallel to each other, see inset of Fig. 3(a). Figure 3(a) shows the imaginary band diagram of a complex SC containing the aforementioned parallel non-Hermiticity with $\beta=0.03$, as calculated by the FEM and PWE method. The real bands remain identical to the Hermitian system. Since the gain and loss cylinders are parallel to the $M X$ direction, complex conjugate eigenmodes will form only along this path. Figure 3(b) displays a zoom of the upper CSC corner, depicting how the interface between the trivial and nontrivial regions (gain and loss cylinders are interchanged in the regions), is formed. Accordingly, the left (right) hinge will host gain (loss) cylinders. The complex eigenmodes depicted in Figs. 3(c) and 3(d), display, similar to the Hermitian case, four pseudohinge and four degenerate corner states. While the pseudohinge states $\mathrm{pH}_{1}$ and $\mathrm{pH}_{4}$ remain purely real, $\mathrm{pH}_{2}$ and $\mathrm{pH}_{3}$ appear as a complex conjugate pair. Similar to the latter two states, all cornerconfined modes, as rendered in Figs. 3(c) and 3(d), exist in the form of two pairs of complex conjugate corner states. Their corresponding absolute pressure eigenmode profiles are illustrated in Figs. 3(e) and 3(f). The purely real pseudohinge states confine similar as in Hermitian CSCs. The states $\mathrm{pH}_{2}\left(\mathrm{pH}_{3}\right)$ on the other hand, confine along the left (right) hinge, at which they constitute an amplifying acoustic source (attenuating acoustic sink) thanks to their negative (positive) imaginary eigenfrequency [57]. Further, we discuss the influence of the crystal termination on the pseudohinge states [57]. Concerning the corner states, as shown in Fig. 3(f), as opposed to the Hermitian CSC sustaining (off-) diagonal and four simultaneously excited corner states, we now have them confined in a similar fashion to the complex hinge states. The states confining at the right-top and right-bottom corners $\left(C_{1}\right.$ and $\left.C_{3}\right)$ have positive imaginary frequencies
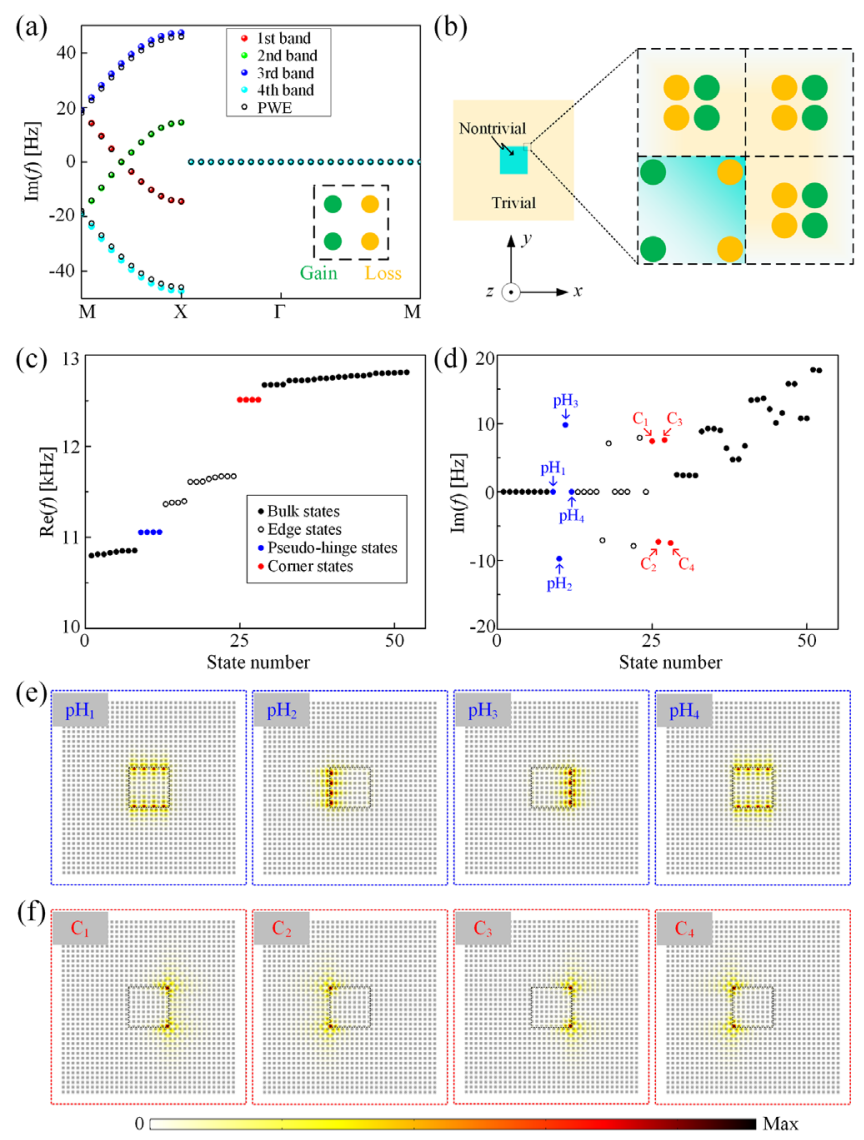

FIG. 3. CSC with parallel non-Hermiticity. (a) Imaginary band diagram of the sonic modes in the presence of parallel nonHermiticity. Colored curves display the results calculated with the FEM and the circles represent the bands obtained by the PWE method. Inset: the metamolecule with parallel arrangement of gain and loss components. (b) Zoom of the CSC displaying the arrangement of the trivial and nontrivial regions. Simulated (c) real and (d) imaginary eigenfrequencies for the parallel non-Hermiticity CSC. The eigenfrequencies of the second $\left(\mathrm{pH}_{2}\right)$ and third $\left(\mathrm{pH}_{3}\right)$ pseudohinge states and all four corner states $\left(C_{1}, C_{2}, C_{3}\right.$, and $C_{4}$ ) become complex conjugate pairs. Acoustic eigenmodes profiles of (e) pseudohinge and (f) corner states.

and are thus attenuating modes, whereas the states at the left-top and left-bottom corners $\left(C_{2}\right.$ and $\left.C_{4}\right)$ have negative imaginary frequencies and are amplifying. It is worth noting that due to inversion symmetry of the CSC, we are able to make the attenuating pseudohinge and corner state amplify and vice versa by changing the sign of $\beta$ while keeping the real eigenvalues unchanged.

The second non-Hermitian arrangement of the CSC comprises diagonally symmetrical gain and loss units as can be seen in Fig. 4. Thus, the imaginary band diagram presented in Fig. 4(a) clearly illustrates that the only nonzero but complex conjugate imaginary eigenmodes appear along the $\Gamma M$ direction, which is symmetric to the diagonal non-Hermiticity axis of the metamolecule. Again, the bands calculated by FEM and PWE with $\beta=$ 0.03 display excellent agreement. Based on this new 

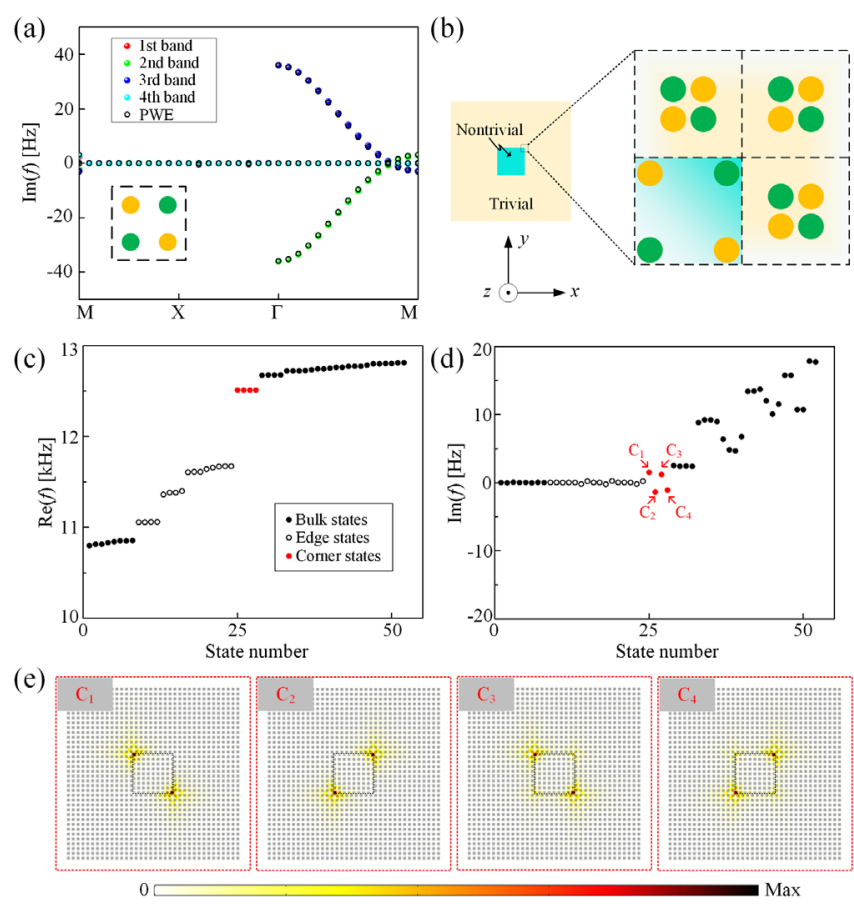

FIG. 4. CSC with diagonal non-Hermiticity. (a) Imaginary dispersion relations. (b) Enlargement of the CSC displaying the arrangement of the trivial and nontrivial regions. Simulated (c) real and (d) imaginary eigenfrequencies for the diagonal nonHermiticity crystal. The eigenfrequencies of four corner states $\left(C_{1}, C_{2}, C_{3}\right.$, and $\left.C_{4}\right)$ become complex conjugate. (e) Acoustic eigenmode profile of corner states.

symmetry, we construct a new CSC containing diagonal non-Hermiticity metamolecules as displayed in Fig. 4(b). The real and imaginary frequencies of the eigenmodes of this crystal that are illustrated in Figs. 4(c) and 4(d), show the disappearance of the pseudohinge states but display that four degenerate corner states still exist. Unlike the previously discussed corner states sustained in the parallel non-Hermiticity CSC, which confine at two corners of lossy or attenuating hinge, the diagonal non-Hermiticity CSC hosts remarkably different corner states. As shown in Fig. 4(e), the states $C_{1}$ and $C_{3}$ that behave like sinks as of the positive imaginary frequency, bind at the diagonal corners as opposed to the $C_{2}$ and $C_{4}$ source states that act as sound amplifiers at the off-diagonal corners of the nonHermitian CSC.

To shed more light on the non-Hermitian HOTIs and their unusual characteristics we embark on the analysis of the $\mathcal{P} \mathcal{T}$ symmetry phases. The complex phase diagram of a CSC containing parallel non-Hermitian metamolecules is plotted in Figs. 5(a) and 5(b). We immediately observe that the spectral location of the real part of the pseudohinge states $\mathrm{pH}_{2} / \mathrm{pH}_{3}$ and the corner states $C_{1} / C_{2}$ remain unaffected with a growing non-Hermiticity parameter $\beta$. Their imaginary counterparts clearly display a thresholdless phase transition as the complex conjugate states grow
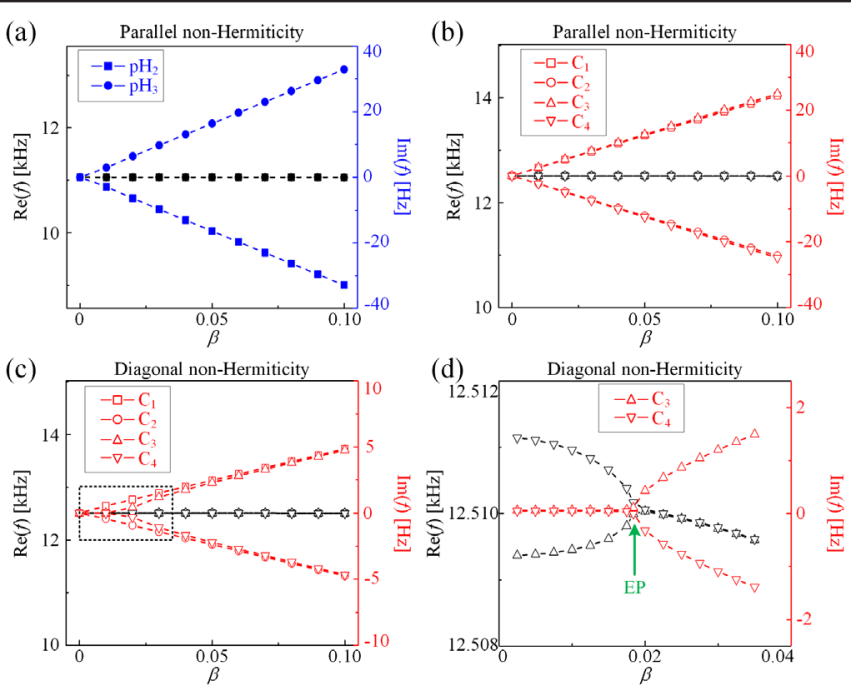

FIG. 5. $\mathcal{P} \mathcal{T}$-symmetry phase diagram. The complex (a) pseudohinge and (b) corner states with parallel non-Hermiticity, and (c) the corner states with diagonal non-Hermiticity are calculated with varying $\beta$. (d) Enlarged view of the $\mathcal{P} \mathcal{T}$-symmetry breaking transition of $C_{3}$ and $C_{4}$ within the black dashed frame in (c).

linearly with $\beta$ within the $\mathcal{P} \mathcal{T}$-symmetry broken region. Interestingly, when computing the complex phase diagram for a CSC containing diagonal non-Hermitian metamolecules, we predict the onset of a state-selective $\mathcal{P} \mathcal{T}$ symmetry breaking transition. In the absence of pseudohinge states in this scenario, in Figs. 5(c) and 5(d) the complex corner states $C_{1} / C_{2}$ and $C_{3} / C_{4}$ are computed as a function of $\beta$. The complex conjugate states $C_{1} / C_{2}$, similar to the states in the parallel non-Hermiticity CSC, grow linearly with $\beta$ within the thresholdless broken $\mathcal{P} \mathcal{T}$ symmetry phase. However, when zooming into Fig. 5(c) as displayed in Fig. 5(d) we identify the coalescence of the $C_{3}$ and $C_{4}$ corner states at about $\beta=0.0184$. This transition point is marked as the exceptional point, around which, for $\beta<0.0184$ the two acoustic corner states reside within $\mathcal{P} \mathcal{T}$-symmetric phase at which the real eigenfrequencies are nondegenerate, while for $\beta>0.0184$, within the $\mathcal{P} \mathcal{T}$ broken phase, the real part degenerates while the zero imaginary components transition into a complex conjugate pair. This phase transition is accompanied by four simultaneously excited real corner states converting into attenuating (amplifying) diagonal (off-diagonal) corner states [57]. Finally, we emphasize that these complex corner states are protected by pseudo-anti-Hermiticity at which the real (imaginary) states appear gapless (gapped) [64].

In conclusion, we have demonstrated acoustic secondorder topological insulators and their topological pseudohinge and corner states both in Hermitian and non-Hermitian systems. Depending on the geometrical implementation of the non-Hermiticity within the crystals, the pseudohinge and the corner states can be tuned to be either attenuating or amplifying. Part of the acoustic modes reside always within 
the $\mathcal{P} \mathcal{T}$-broken phase without the presence of a transition threshold; however, we have found an exceptional point marking the evolution of real corner states into diagonal and off-diagonal complex corner states. Beyond the academic importance of our findings, we foresee new possibilities for sonic energy focusing and controlling sound propagations in non-Hermitian systems for broadened acoustic device functionalities.

We gratefully acknowledge fruitful discussions with Feng Liu (Kwansei Gakuin University). This work was supported by National Key R\&D Program of China (2017YFA0303702), NSFC (11834008, 11874215, 11674172, and 11574148), Jiangsu Provincial NSF (BK20160018), the Fundamental Research Funds for the Central Universities (020414380001) and Nanjing University Innovation and Creative Program for $\mathrm{PhD}$ candidate (CXCY17-11). Z.Z. acknowledges the support from the China Scholarship Council. J.C. acknowledges the support from the European Research Council (ERC) through the Starting Grant No. 714577 PHONOMETA and from the MINECO through a Ramón y Cajal grant (Grant No. RYC2015-17156).

Note added in the proof.-We became aware of a recently published paper where single corner zero-energy modes localization in a 2D non-Hermitian SOTI was discussed [65].

*chengying@nju.edu.cn

†liuxiaojun@nju.edu.cn

\#johan.christensen@uc3m.es

[1] M. Z. Hasan and C. L. Kane, Rev. Mod. Phys. 82, 3045 (2010).

[2] X.-L. Qi and S.-C. Zhang, Rev. Mod. Phys. 83, 1057 (2011).

[3] A. Bansil, H. Lin, and T. Das, Rev. Mod. Phys. 88, 021004 (2016).

[4] F. D. M. Haldane and S. Raghu, Phys. Rev. Lett. 100, 013904 (2008).

[5] Z. Wang, Y. Chong, J. D. Joannopoulos, and M. Soljačić, Nature (London) 461, 772 (2009).

[6] M. Hafezi, E. A. Demler, M. D. Lukin, and J. M. Taylor, Nat. Phys. 7, 907 (2011).

[7] Y. Poo, R.-x. Wu, Z. Lin, Y. Yang, and C. T. Chan, Phys. Rev. Lett. 106, 093903 (2011).

[8] A. B. Khanikaev, S. Hossein Mousavi, W.-K. Tse, M. Kargarian, A. H. MacDonald, and G. Shvets, Nat. Mater. 12, 233 (2013).

[9] M. C. Rechtsman, J. M. Zeuner, Y. Plotnik, Y. Lumer, D. Podolsky, F. Dreisow, S. Nolte, M. Segev, and A. Szameit, Nature (London) 496, 196 (2013).

[10] W.-J. Chen, S.-J. Jiang, X.-D. Chen, B. Zhu, L. Zhou, J.-W. Dong, and C. T. Chan, Nat. Commun. 5, 5782 (2014).

[11] L. Lu, J. D. Joannopoulos, and M. Soljačić, Nat. Photonics 8, 821 (2014).
[12] L.-H. Wu and X. Hu, Phys. Rev. Lett. 114, 223901 (2015).

[13] J.-W. Dong, X.-D. Chen, H. Zhu, Y. Wang, and X. Zhang, Nat. Mater. 16, 298 (2017).

[14] A. B. Khanikaev and G. Shvets, Nat. Photonics 11, 763 (2017).

[15] F. Gao, H. Xue, Z. Yang, K. Lai, Y. Yu, X. Lin, Y. Chong, G. Shvets, and B. Zhang, Nat. Phys. 14, 140 (2018).

[16] Z. Yang, F. Gao, X. Shi, X. Lin, Z. Gao, Y. Chong, and B. Zhang, Phys. Rev. Lett. 114, 114301 (2015).

[17] A. B. Khanikaev, R. Fleury, S. H. Mousavi, and A. Alù, Nat. Commun. 6, 8260 (2015).

[18] M. Xiao, W.-J. Chen, W.-Y. He, and C. T. Chan, Nat. Phys. 11, 920 (2015).

[19] J. Lu, C. Qiu, L. Ye, X. Fan, M. Ke, F. Zhang, and Z. Liu, Nat. Phys. 13, 369 (2017).

[20] Z. Zhang, Q. Wei, Y. Cheng, T. Zhang, D. Wu, and X. Liu, Phys. Rev. Lett. 118, 084303 (2017).

[21] Z. Zhang, Y. Tian, Y. Cheng, X. Liu, and J. Christensen, Phys. Rev. B 96, 241306(R) (2017).

[22] F. Li, X. Huang, J. Lu, J. Ma, and Z. Liu, Nat. Phys. 14, 30 (2018).

[23] J. Lu, C. Qiu, W. Deng, X. Huang, F. Li, F. Zhang, S. Chen, and Z. Liu, Phys. Rev. Lett. 120, 116802 (2018).

[24] H. He, C. Qiu, L. Ye, X. Cai, X. Fan, M. Ke, F. Zhang, and Z. Liu, Nature (London) 560, 61 (2018).

[25] Z. Zhang, Y. Tian, Y. Cheng, Q. Wei, X. Liu, and J. Christensen, Phys. Rev. Applied 9, 034032 (2018).

[26] Z. Zhang, Y. Tian, Y. Wang, S. Gao, Y. Cheng, X. Liu, and J. Christensen, Adv. Mater. 30, 1803229 (2018).

[27] X. Zhang, M. Xiao, Y. Cheng, M.-H. Lu, and J. Christensen, Commun. Phys. 1, 97 (2018).

[28] R. Süsstrunk and S. D. Huber, Science 349, 47 (2015).

[29] S. H. Mousavi, A. B. Khanikaev, and Z. Wang, Nat. Commun. 6, 8682 (2015).

[30] M. Miniaci, R. K. Pal, B. Morvan, and M. Ruzzene, Phys. Rev. X 8, 031074 (2018).

[31] W. A. Benalcazar, B. A. Bernevig, and T. L. Hughes, Science 357, 61 (2017).

[32] J. Langbehn, Y. Peng, L. Trifunovic, F. von Oppen, and P. W. Brouwer, Phys. Rev. Lett. 119, 246401 (2017).

[33] Z. Song, Z. Fang, and C. Fang, Phys. Rev. Lett. 119, 246402 (2017).

[34] W. A. Benalcazar, B. A. Bernevig, and T. L. Hughes, Phys. Rev. B 96, 245115 (2017).

[35] M. Ezawa, Phys. Rev. Lett. 120, 026801 (2018).

[36] F. Schindler, A. M. Cook, M. G. Vergniory, Z. Wang, S. S. P. Parkin, B. A. Bernevig, and T. Neupert, Sci. Adv. 4, eaat0346 (2018).

[37] B. Y. Xie, H. F. Wang, H. X. Wang, X. Y. Zhu, J. H. Jiang, M. H. Lu, and Y. F. Chen, Phys. Rev. B 98, 205147 (2018).

[38] M. Serra-Garcia, V. Peri, R. Süsstrunk, O. R. Bilal, T. Larsen, L. G. Villanueva, and S. D. Huber, Nature (London) 555, 342 (2018).

[39] C. W. Peterson, W. A. Benalcazar, T. L. Hughes, and G. Bahl, Nature (London) 555, 346 (2018).

[40] S. Imhof et al., Nat. Phys. 14, 925 (2018).

[41] H. Xue, Y. Yang, F. Gao, Y. Chong, and B. Zhang, Nat. Mater. 18, 108 (2019).

[42] X. Ni, M. Weiner, A. Alù, and A. B. Khanikaev, Nat. Mater. 18, 113 (2019). 
[43] I. Y. Goldsheid and B. A. Khoruzhenko, Phys. Rev. Lett. 80, 2897 (1998).

[44] C. M. Bender, D. C. Brody, and H. F. Jones, Phys. Rev. Lett. 89, 270401 (2002).

[45] C. Dembowski, B. Dietz, H. D. Gräf, H. L. Harney, A. Heine, W. D. Heiss, and A. Richter, Phys. Rev. Lett. 90, 034101 (2003).

[46] C. M. Bender, D. C. Brody, and H. F. Jones, Phys. Rev. D 70, 025001 (2004).

[47] K. G. Makris, R. El-Ganainy, D. N. Christodoulides, and Z.H. Musslimani, Phys. Rev. Lett. 100, 103904 (2008).

[48] Y. D. Chong, L. Ge, H. Cao, and A. D. Stone, Phys. Rev. Lett. 105, 053901 (2010).

[49] C. E. Rüter, K. G. Makris, R. El-Ganainy, D. N. Christodoulides, M. Segev, and D. Kip, Nat. Phys. 6, 192 (2010).

[50] A. Szameit, M. C. Rechtsman, O. Bahat-Treidel, and M. Segev, Phys. Rev. A 84, 021806(R) (2011).

[51] B.-I. Popa and S. A. Cummer, Nat. Commun. 5, 3398 (2014).

[52] R. Fleury, D. Sounas, and A. Alù, Nat. Commun. 6, 5905 (2015).

[53] J. Christensen, M. Willatzen, V. R. Velasco, and M. H. Lu, Phys. Rev. Lett. 116, 207601 (2016).

[54] Y. Li, C. Shen, Y. Xie, J. Li, W. Wang, S. A. Cummer, and Y. Jing, Phys. Rev. Lett. 119, 035501 (2017).
[55] T. Liu, X. Zhu, F. Chen, S. Liang, and J. Zhu, Phys. Rev. Lett. 120, 124502 (2018).

[56] M. Rosendo-López, A. Merkel, and J. Christensen, Europhys. Lett. 124, 34001 (2018).

[57] See Supplemental Material at http://link.aps.org/ supplemental/10.1103/PhysRevLett.122.195501for a discussion on the detailed derivation of the PWE method, topological phase transition with varying the ratio $D / a$, comparison between the conventional edge states and the pseudohinge states, dispersion relation of 1D ribbon shaped non-Hermitian structure, dependence of the pseudohinge states on the crystal termination, and eigenmodes of corner states within the exact and broken $\mathcal{P} \mathcal{T}$-symmetric phase.

[58] F. Liu, H.-Y. Deng, and K. Wakabayashi, Phys. Rev. B 97, 035442 (2018).

[59] C. Fang, M. J. Gilbert, and B. A. Bernevig, Phys. Rev. B 86, 115112 (2012).

[60] F. Liu and K. Wakabayashi, Phys. Rev. Lett. 118, 076803 (2017).

[61] J. Zak, Phys. Rev. Lett. 62, 2747 (1989).

[62] Y. Ota, F. Liu, R. Katsumi, K. Watanabe, K. Wakabayashi, Y. Arakawa, and S. Iwamoto, arXiv:1812.10171.

[63] F. Liu, H.-Y. Deng, and K. Wakabayashi, Phys. Rev. Lett. 122, 086804 (2019).

[64] S. Lieu, Phys. Rev. B 97, 045106 (2018).

[65] T. Liu, Y. R. Zhang, Q. Ai, Z. Gong, K. Kawabata, M. Ueda, and F. Nori, Phys. Rev. Lett. 122, 076801 (2019). 